\title{
The GMI-Hysteresis of $\mathrm{Co}_{67} \mathrm{Fe}_{4.5} \mathrm{Cu}_{1} \mathrm{Si}_{12.5} \mathrm{~B}_{15}$ Amorphous Thin Strip
}

\author{
Jie Hao ${ }^{1,2, a}$, Jinhui Huang ${ }^{1}$, Ang Ding ${ }^{1}$, Kunming Qian ${ }^{1}$, Yansong Zhang ${ }^{1}$, \\ Mingli $\mathrm{Li}^{1}$, Song $\mathrm{Ji}^{1}$, Mi Yan \\ ${ }^{1}$ Ningbo Branch of China Academy Ordnance Science, No. 199 Lingyun Road, Ningbo, 315103, \\ China \\ ${ }^{2}$ Department of Material Science and Engineering, State Key Laboratory of Silicon Materials, \\ Zhejiang University, No. 38 Zheda Road, Hangzhou, 310027, China \\ ahao_jie82@163.com
}

Keywords:GMI; amorphous thin strip; GMI-hysteresis; driving current; driving frequenc

Abstract. This paper presents the results of investigations recently done by our research group that improve the properties stability of GMI sensors by exploring the giant magneto-impedance (GMI) and the GMI-hysteresis of $\mathrm{Co}_{67} \mathrm{Fe}_{4.5} \mathrm{Cu}_{1} \mathrm{Si}_{12.5} \mathrm{~B}_{15}$ amorphous thin strips, which was the most promising soft magnetic material could be used in the weak magnetic transducers. The amorphous thin strips were prepared by the so-called planar flow casting technology. With the circumferential driving mode, the changes of the GMI on the frequency of the ac current under different external magnetic field were measured on Agilent 4294A precision impedance analyzer. The maximum GMI ratio of $201.4 \%$ was observed. The field sensitivity, $(\delta \mathrm{Z} / \mathrm{Z}) / \delta \mathrm{H}$, has reached $413.2 \%$. Additionally, the ratio of GMI changes of the $\mathrm{CoFeCuSiB}$ amorphous thin strips could be control under $1 \%$, by the optimum parameters of this amorphous strip, such as the DC current and the time of joule current annealing, the dc level and frequency of the excitation current, the driving method.

\section{Introduction}

The giant magneto-impedance (GMI) effect arises from the change of the high frequency ac permeability of magnetic material submitted to the change of external magnetic field[1, 2]. It has attracted much attention of scholar and scientist for two decades, mainly because of its potential for high sensitivity magnetic transducers[3,4]. The Co-base amorphous material has been used in geomagnetism sensor for its low coercivity $\left(\mathrm{H}_{\mathrm{c}}\right)$, high saturation magnetization $\left(\mathrm{B}_{\mathrm{s}}\right)$, large permeability, and nearly zero magnetostriction[5-8]. As yet the development of weak magnetic sensor was obstructed by the GMI-hysteresis of Co-base amorphous material, herein called as "the ratio of GMI changes of Co-base amorphous material". The GMI-hysteresis was defined as the reversible relaxation of the low-field magneto-impedance after the direction change of the external magnetic field $\left(\mathrm{H}_{\mathrm{ex}}\right)$.

In this paper, the influences of the treat time and DC current of annealing, the dc level and frequency of the excitation current, the driving method on GMI effect and GMI-hysteresis of Co-base amorphous thin strips were in-depth investigated on the basis of our previous experimental results[9]. With the circumferential driving mode, we studied the influence of the DC current and the time of annealing on the complex impedance of the $\mathrm{Co}_{67} \mathrm{Fe}_{4.5} \mathrm{Cu}_{1} \mathrm{Si}_{12.5} \mathrm{~B}_{15}$ amorphous thin strip, and investigated the GMI effect and GMI-hysteresis of the complex impedance after a change in the magnetic field direction. Through the existing experiment we can find out the optimum parameters of the amorphous strip to improving the stability and accuracy of the material for technical applications in the weak magnetic sensors.

\section{Experimental details}

Cast ingots alloy with nominal composition (in at. \%) Co67, Fe4.5, Cu1, Si12.5, B15 were obtained by arc melting the metals $\mathrm{Co}, \mathrm{Cu}, \mathrm{Fe}-\mathrm{Si}, \mathrm{Fe}-\mathrm{B}$ (Co, Fe, Cu, Fe-B 99.9\%, Fe-Si99.9\%) under high purity Ar atmosphere. Then the Co-base amorphous thin strips, with dimensions of $20 \mathrm{~m} \times 100 \mu \mathrm{m} \times 20$ $\mu \mathrm{m}$, were fabricated from the cast ingots alloy by planar flow casting in air on the copper wheel of 
$300 \mathrm{~mm}$ diameter. And next the amorphous thin strips were submitted to different thermal treatment in order to release the stress and induce transverse anisotropy in the strips. In here, we adopted three kinds of joule current annealing process to treat the cast strips, respectively, such as the sample A was treated with the process A by current value: $250 \mathrm{~mA}$, and annealing time: $100 \mathrm{~s}$, and the sample B was treated with the process B by current value: $300 \mathrm{~mA}$, and annealing time: $100 \mathrm{~s}$, and the sample $\mathrm{C}$ was treated with the process $\mathrm{C}$ by current value: $250 \mathrm{~mA}$, and annealing time: $150 \mathrm{~s}$. And then with the circumferential driving mode, the GMI properties of all treated samples with dimensions of 40 $\mathrm{mm} \times 100 \mu \mathrm{m} \times 20 \mu \mathrm{m}$ were measured by Agilent4294A precision impedance analyzer with four-terminal method under different external magnetic field of 0-50 Oe. During the measurement, the direction of the ac current and the external magnetic field was along the longitudinal direction of the strips, and the frequency of the ac current flown through the usual sensors ranges from $0.1-10$ $\mathrm{MHz}$ with a constant current amplitude of $10 \mathrm{~mA}$. Above measurements were carried out at room temperature and without geomagnetism disturbance.

\section{Results and discussion}

3.1 The Relations between Impedance and measurement parameter

Frequency dependence of impedance for $\mathrm{Co}_{67} \mathrm{Fe}_{4.5} \mathrm{Cu}_{1} \mathrm{Si}_{12.5} \mathrm{~B}_{15}$ amorphous thin strips under different external magnetic field were measured with a $10 \mathrm{~mA}$ ac exciting current in the frequency range 100 KHz-10 MHz. The typical impedance test result of amorphous thin strips was shown in Fig. 1. From the figure, it was clear that on increasing the exciting frequency the impedance increases steadily in the external magnetic field 0-50 Oe. The phenomenon was attributed to the change of skin depth[10-12], which was closely correlated with the exciting frequency. We could observe a mutation of impedance occurred around $5 \mathrm{MHz}$, some researchers consider which were induced by thermal magnetic resonance, but some other researchers think which were induced by the inherent characteristics of the measuring equipments. Compared with the relation between the impedance and the exciting frequency, the $\mathrm{H}_{\mathrm{ex}}$ dependence of impedance for the amorphous thin strips shown a completely different result. With an increase of the external magnetic fields, the increase ratio of the impedance increased obviously at low frequency of $100 \mathrm{kHz}-4 \mathrm{MHz}$, and reached a max value at 0.9 Oe, then decreased steadily. This may be caused by an increase in the average susceptibility at low $\mathrm{H}_{\mathrm{ex}}$ values as we align the magnetization axially. At high enough axial field values a pure resistive behavior is expected, the magneto-impedance is then saturated. These results indicated that, at a fixed exciting frequency, there existed an optical $\mathrm{H}_{\mathrm{ex}}$ at which the impedance of amorphous thin strips could reach the max value. From the Fig. 1 , it could be found that the optical $\mathrm{H}_{\mathrm{ex}}$ will increase with the increase of the exciting frequency. In the next section, this phenomenon will discuss in detail combined with the following experiments and analyse results.

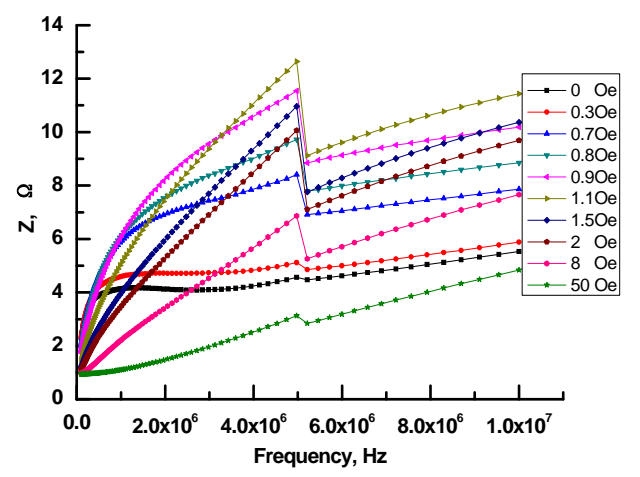

Figure 1. Frequency dependence of impedance for $\mathrm{Co}_{67} \mathrm{Fe}_{4.5} \mathrm{Cu}_{1} \mathrm{Si}_{12.5} \mathrm{~B}_{15}$ amorphous thin strips under different external magnetic field (0-50 Oe). 


\subsection{The GMI properties of the CoFeCuSiB Strip}

\subsubsection{The impedance $(Z)$ of the CoFeCuSiB Strip}

According to the actual application requirements of magnetic sensor, the impedance change of the three treated samples must be tested in the magnetization and reversal-magnetization processes (herein called as "negative and positive magnetic fields") at the exciting frequency $\mathrm{f}=1 \mathrm{MHz}, 3 \mathrm{MHz}$, $5 \mathrm{MHz}, 10 \mathrm{MHz}$, and the results were shown in the Fig.2. All samples had shown a similar change tendency and law as be seen from the Fig.2. With the increase of the $\mathrm{H}_{\mathrm{ex}}$, the impedance of the strips fist increased to the max, and then decreased monotonically with further increase of external fields. So all curve shown double peaks in negative and positive magnetic fields. The $\mathrm{Z}$ peak was mainly attributed to the dynamic magnetization process where the dominating magnetization process varies from domain wall movement at relatively low frequency to the magnetization rotation at relatively high frequency $[10,13,14]$. The $\mathrm{Z}_{0}\left(\mathrm{H}_{\mathrm{ex}}=0\right)$ increased obviously with increasing exciting frequency, and became more pronounced for $\mathrm{f}=10 \mathrm{MHz}$. The phenomenon was attributed to the imaginary of impedance, namely the reactance, which increase rapidly at high frequency. At the same time, the external fields of $Z_{\max }$ also shifted to a more strong external fields with the increase of the exciting frequency, such as the sample $A$, the external fields of $Z_{\max }$ shifted from $0.9 \mathrm{Oe}$ at $1 \mathrm{MHz}$ to 1.1 Oe at $10 \mathrm{MHz}$ in the positive magnetic fields, and from $-0.8 \mathrm{Oe}$ at $1 \mathrm{MHz}$ to $-1.0 \mathrm{Oe}$ at $10 \mathrm{MHz}$ in the negative magnetic fields, it should be further studied and explored to find out the inner relationship between them through the magneto-electric effect. It also could be found in the curves, with an increase of the negative and positive $\mathrm{H}_{\mathrm{ex}}$, all curves display a relative symmetry, the symmetry were improved steadily with decrease the exciting frequency, especially for the $\mathrm{Z}$ curve at low frequency of $1 \mathrm{MHz}$. This suggests that the low exciting frequency enables the strips to take on a broader working range for weak magnetic field detection.
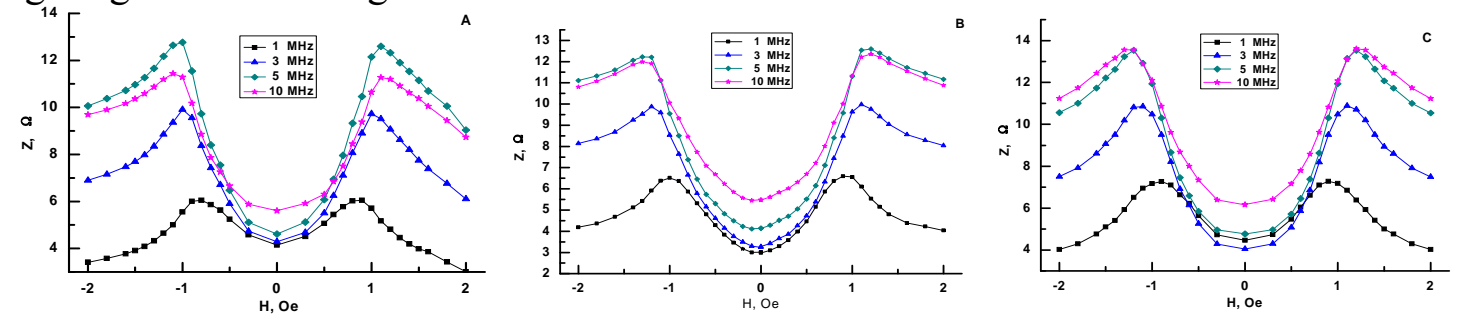

Figure 2. The Z-hysteresis of samples treated by various joule-heating conditions, as a function of applied field measured at frequency $\mathrm{f}=1 \mathrm{MHz}, 3 \mathrm{MHz}, 5 \mathrm{MHz}, 10 \mathrm{MHz}$, indicated in the figure: (A) $250 \mathrm{~mA}, 100 \mathrm{~s}$, (B) $300 \mathrm{~mA}, 100 \mathrm{~s},(\mathrm{C}) 250 \mathrm{~mA}, 150 \mathrm{~s}$.

\subsubsection{The $\delta Z / Z$ of the CoFeCuSiB Strip}

From the data of Fig. 2, the magneto-impedance ratio $\delta Z / Z$ can been calculated by the formula: $\delta Z / Z(100 \%)=[Z(H)-Z(H=0)] / Z(H=0)$, and the calculated results were shown in the Fig. 3. In general, it can be seen in Fig. 3 that at $f=1 \mathrm{MHz}$ the $\delta \mathrm{Z} / \mathrm{Z}$ of the samples almost had a little of response to an external magnetic field. Meanwhile, at $\mathrm{f}=3 \mathrm{MHz}, 5 \mathrm{MHz}$ and $10 \mathrm{MHz}$, the sample exhibit the GMI effects and a double-peak feature was observed in the GMI curves at all frequency. As the frequency is increased, however, all the three samples show a more obvious GMI effects. With the increase of the $\mathrm{H}_{\mathrm{ex}}$ or the exciting frequency, the $\delta \mathrm{Z} / \mathrm{Z}$ of the strips also fist increased to the max, and then decreased monotonically with further increase of external fields or the frequency keeping to the same change style with the impedance. This finding result could be explained by considering the relative contributions of the permeability and the skin depth. The application of $\mathrm{H}_{\mathrm{ex}}$ along the strips would suppress the transverse permeability (perpendicular direction to the axis of strip), which in turn results in an increase of skin depth and consequently yields the large variation of the impedance, and hence to increase GMI effect $[15,16]$.

As expected from changes of the $\delta Z / Z$ (Fig.3), significant differences in dependences for different annealed samples were observed. The $\delta Z / Z$ ratio of the sample $A$ in positive and negative magnetic fields, which was treated with $250 \mathrm{~mA}$ current and $100 \mathrm{~s}$ annealing time, increased from $46.1 \%$ and $46.0 \%$ at $1 \mathrm{MHz}$ to $181.3 \%$ and $173.2 \%$ at $5 \mathrm{MHz}$, and with further increasing the exciting 
frequency, which reduced to $106.2 \%$ and $101.6 \%$ at $10 \mathrm{MHz}$, respectively. With increase the treat current to $300 \mathrm{~mA}$, the $\delta \mathrm{Z} / \mathrm{Z}$ ratio of the sample $\mathrm{B}$ in negative and positive magnetic fields increased from $113.2 \%$ and $108.6 \%$ at $1 \mathrm{MHz}$ to $201.4 \%$ and $192.2 \%$ at $5 \mathrm{MHz}$, respectively, and then decreased to $119.5 \%$ and $120.7 \%$ at $10 \mathrm{MHz}$. Based on the treat process $\mathrm{A}$, the treat time was prolonged by $50 \mathrm{~s}$, the $\delta \mathrm{Z} / \mathrm{Z}$ ratio of the sample $\mathrm{C}$ in positive magnetic fields was improved slightly and only increased from $46.1 \%$ of sample A to $60.0 \%$ of sample $\mathrm{C}$ at $1 \mathrm{MHz}$, and from $181.3 \%$ of sample A to $185.5 \%$ of sample $\mathrm{C}$ at $5 \mathrm{MHz}$. It is worth mentioning, that the change of $\delta \mathrm{Z} / \mathrm{Z}$ were attributed to magnetic anisotropy field, which were changed by annealing temperature, namely by value of the treat current. Increasing of the annealing current results in increase of $\delta Z / Z$ and decrease of magnetic anisotropy field. These experiments found that field dependence of the $\delta Z / Z$ in the joule current annealed $\mathrm{CoFeCuSiB}$ in the air was asymmetric two-peak, with the peak at the positive field being higher than the peak at the negative field. The reason was that the annealed ribbon consists of the amorphous soft magnetic layer and two surface hard magnetic crystalline layers with an effective unidirectional anisotropy, and it affected the magnetization variations.
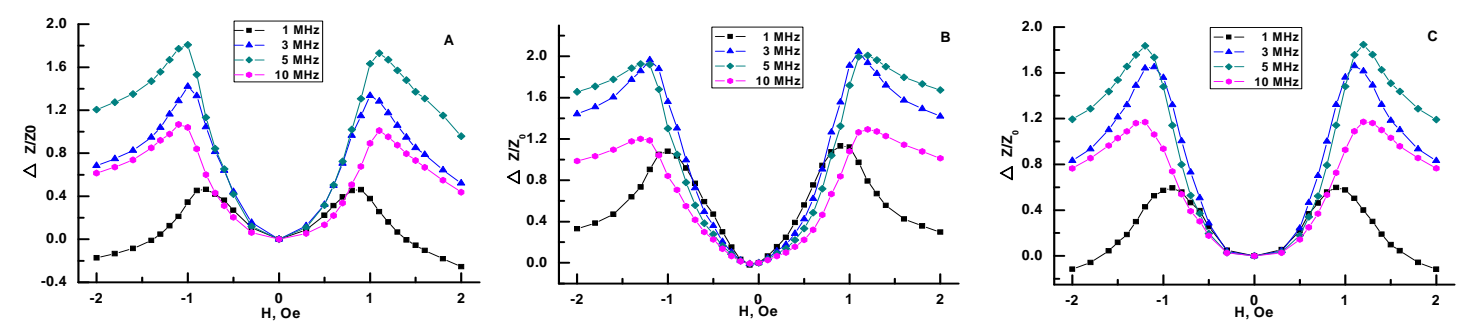

Figure 3. The $\delta Z / Z$-hysteresis, as a function of applied field measured at frequency $\mathrm{f}=1 \mathrm{MHz}, 3 \mathrm{MHz}$, $5 \mathrm{MHz}, 10 \mathrm{MHz}$ for samples with various Joule-heating conditions indicated in the figure: (A) 250 $\mathrm{mA}, 100 \mathrm{~s}$; (B) $260 \mathrm{~mA}, 110 \mathrm{~s}$; (C) $260 \mathrm{~mA}, 100 \mathrm{~s}$.

\subsubsection{The field sensitivity of the CoFeCuSiB Strip}

For weak magnetic sensors, the magnetic sensitivity of CoFeCuSiB Strip was very important parameter. From the $\delta Z / Z$ curves (Fig.3), the field sensitivity (S) of the strips were calculated by the formula: $\mathrm{S}=[\mathrm{d}(\delta \mathrm{Z} / \mathrm{Z}) / \mathrm{dH} \times 100 \%$, and the calculated results were shown in the Fig.4. It could be seen in Fig. 4 that the sample exhibited a giant field sensitivity and a double-peak feature at all frequency, which were similar to the $\delta \mathrm{Z} / \mathrm{Z}$. With the increase of the $\mathrm{H}_{\mathrm{ex}}$ or the exciting frequency, the magnetic sensitivity of the strips also fist increased to the max, and then decreased monotonically with further increase of them. As similar to the $\delta \mathrm{Z} / \mathrm{Z}$, the $\mathrm{S}$ of the sample A in positive and negative magnetic fields increased from $91.1 \%$ and $92.8 \%$ at $1 \mathrm{MHz}$ to $386.6 \%$ and $403.3 \%$ at $5 \mathrm{MHz}$, and then declined to $242.5 \%$ and $247.2 \%$ at $10 \mathrm{MHz}$, respectively. Similarly, the sample B and C had the same change trend with the sample A. They all exhibited asymmetry, except for the samples C. The max field sensitivity of the sample A had reached $403.3 \% / O e$ in negative magnetic fields at $5 \mathrm{MHz}$, but that of the sample $\mathrm{B}$ and the sample $\mathrm{C}$ had reached $411.9 \% / \mathrm{Oe}$ and $357.1 \% / \mathrm{Oe}$ in negative magnetic fields at $5 \mathrm{MHz}$, respectively. So the current value and the treat time affecting on the GMI effect were very different. From the results of the sample A and B, increasing the current value could increase the impedance rate and magnetic field sensitivity of the strip, but the effect of treat time to the properties was not obvious. The above results indicated that the increase of the current value could significantly improved the field sensitivity of strip, but the increase of the treat time could obviously improved the magnetic field sensitivity symmetry of the strips. 

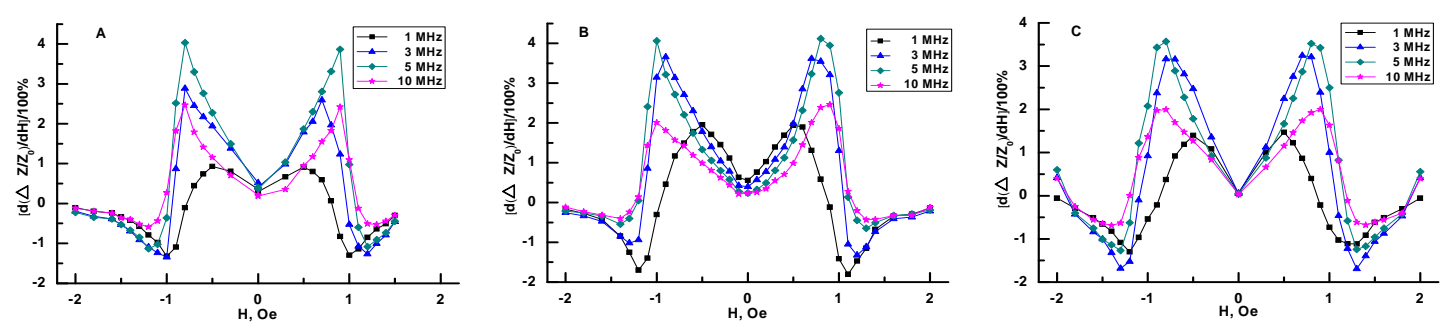

Figure 4. The GMI sensitivity, $\mathrm{d} \delta \mathrm{Z} / \mathrm{Z} 0 / \mathrm{dH} / 100 \%$, as a function of applied field measured at frequency $\mathrm{f}=1 \mathrm{MHz}, 3 \mathrm{MHz}, 5 \mathrm{MHz}, 10 \mathrm{MHz}$ for samples with various Joule-heating conditions indicated in the figure: (A) $250 \mathrm{~mA}, 100 \mathrm{~s}$; (B) $300 \mathrm{~mA}, 100 \mathrm{~s}$; (C) $250 \mathrm{~mA}, 150 \mathrm{~s}$.

\subsection{The GMI-hysteresis of CoFeCuSiB Amorphous Thin Strip}

The GMI hysteresis effect of the amorphous strip refers to the differences between the max rate of change of the impedance at the process of positive magnetization and the max rate of change of the impedance at the process of negative magnetization under the same magnetic field strength rate. The smaller the difference, the better the stability of the weak magnetic sensors, and induced the result the smaller the numerical deviations, the higher the accuracy of the weak magnetic sensors. The maximum sensitivity of the strips in the different excitation frequency was listed in table 1 . As shown in Table 1, with the change of annealing treat, the differences between the max sensitivity at positive and negative magnetic fields change remarkably. As a whole, the GMI-hysteresis effect of the field sensitivity in sample A is higher than that in sample B. with prolonged the annealing time, the maximum sensitivity deceased, but the GMI-hysteresis effect became weak, and improved the stability of the $\mathrm{CoFeCuSiB}$ strip in the external magnetic field.

Table 1. The maximum sensitivity of strips in the different excitation frequency

\begin{tabular}{lllllll}
\hline \multirow{2}{*}{ No. } & \multirow{2}{*}{ Treat condition } & \multirow{2}{*}{$\mathrm{H}_{\mathrm{ex}}$} & $\mathrm{S}$ & & & \\
& & & $1 \mathrm{MHz}$ & $3 \mathrm{MHz}$ & $5 \mathrm{MHz}$ & $10 \mathrm{MHz}$ \\
\hline \multirow{2}{*}{$\mathrm{A}$} & \multirow{2}{*}{$250 \mathrm{~mA}, 100 \mathrm{~s}$} & + & 91.1 & 259.5 & 386.6 & 242.5 \\
& & - & 92.8 & 288.5 & 403.3 & 247.2 \\
\multirow{3}{*}{$\mathrm{B}$} & \multirow{2}{*}{$300 \mathrm{~mA}, 100 \mathrm{~s}$} & + & 194.1 & 361.9 & 411.9 & 246.2 \\
& & - & 195.4 & 365.6 & 406.3 & 211.5 \\
$\mathrm{C}$ & \multirow{2}{*}{$250 \mathrm{~mA}, 150 \mathrm{~s}$} & + & 146.8 & 324.8 & 352.6 & 199.8 \\
& & - & 139.4 & 316.9 & 357.1 & 199.0 \\
\hline
\end{tabular}

According to the data in Fig. 3 and the formula: the ratio of GMI changes $=\left[\operatorname{Max}\left(\delta Z / Z_{0}\right)_{+H e x}\right.$ $\left.-\operatorname{Max}\left(\delta \mathrm{Z} / \mathrm{Z}_{0}\right)_{-\mathrm{Hex}}\right] /(\delta \mathrm{Z} / \mathrm{Z} 0)_{+\mathrm{Hex}} \times 100 \%[17,18]$, the GMI change ratio of the $\mathrm{CoFeCuSiB}$ strips between in the positive and negative magnetic field were shown in Fig. 5. As you can see from Fig. 5, the max of the GMI change ratio at different exciting frequency was belonged to the sample $\mathrm{B}$, and all exceeded 5\%. From Fig.5, we also could find that the GMI change rate of the sample $\mathrm{C}$ at all exciting frequency did not exceed $1 \%$, and this indicated the sample $\mathrm{C}$ possessed a good symmetry and a little of GMI-hysteresis effect. Although the changing rate of impedance and magnetic field sensitivity the sample $\mathrm{C}$ were the least in three samples, but for the stability of GMI effect, it was also the best. So the applications prospect of sample $\mathrm{C}$ was better than the other two samples. We must particularly note that the field sensitivity of the sample $\mathrm{C}$ at excitation frequency $5 \mathrm{MHz}$ exceed $350 \%$ (as shown in Fig.2 and 3). 


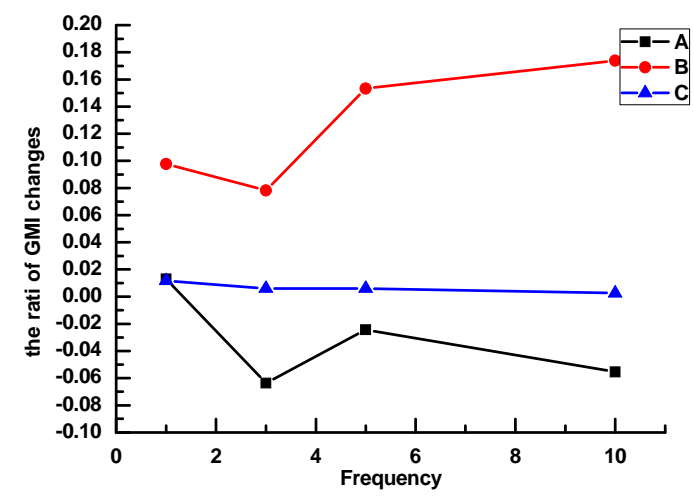

Figure 4. The ratio of GMI changes for the samples at different exciting frequency.

The above results can be interpreted in the following aspects. Firstly, the magnetizations of ferromagnetic materials are controlled by the displacement and rotation process of domain wall and domain, at low exciting frequency, the eddy current damping the displacement of domain wall can be ignored, so the displacement of domain wall was very easily. When the exciting frequencies exceed $\mathrm{MHz}$ level, the eddy current effect become very stronger, and impeded domain wall displacement[19]. And so the magnetization is controlled by the rotation of magnetic moment, in this case, when the external axial magnetic fields increase, the circumferential permeability also increases. The axial magnetic field for magnetic moment of small rotation, so that the tangential permeability increase, and lead to rapid increase of the impedance, the $\delta Z / Z$ and the field sensitivity. Secondly, during the joule current annealing process, the DC current flowing through the wire and producing the circular magnetic field are marked to change the structure of transverse domain in the strips[20], and the magnetization of circular multi-domain system on the transverse axis during magnetization rotation is changing. When the external field is applied, the volume of circular domains of one type increases and that of another type decrease, which induce the change of the impedance. Last, the GMI properties of strip are control by the frequency and the ac current of the exciting current[21]. In order to achieve better application performance of amorphous strips, we must to find an optimal balance point between the DC current and the time of Joule current annealing, the dc level and frequency of the excitation current, the driving method, and so on.

\section{Conclusions}

We studied the GMI effect (dependence of impedance, and GMI ratio) of the CoFeCuSiB strip on applied magnetic field. Joule current annealing process considerable affects the GMI effect of studied the strip. After proper annealing conditions we observed good high GMI effect. We can conclude that we able to control the strip magnetic properties for its application in magnetic sensors through selecting the best parameters of the DC current and the time of joule current annealing, the dc level and frequency of the excitation current, the driving method. The $\mathrm{Co}_{67} \mathrm{Fe}_{4.5} \mathrm{Cu}_{1} \mathrm{Si}_{12.5} \mathrm{~B}_{15}$ strip can present $\mathrm{a} \delta \mathrm{Z} / \mathrm{Z}$ as high as $201.4 \%$ with maximum sensitivity of about $411.9 \% / \mathrm{Oe}$ in annealing at $300 \mathrm{~mA}$ and $100 \mathrm{~s}$. the GMI change rate of the sample C, annealed at $250 \mathrm{~mA}$ and $150 \mathrm{~s}$, did not exceed $1 \%$ at all exciting frequency, and indicated the sample $\mathrm{C}$ possessing a good symmetry and a little of GMI-hysteresis effect, and laid a solid foundation for its further application in micromagnitic sensors.

\section{Acknowledgments}

This work was supported by the Major Project of the National Science and Technology of Ningbo (Grant No. 2011B1013) and the Postdoctoral Science Founfation of China North Materials Science and Engineering Technology Group (Grant No. NBPJ2013-6). We also gratefully acknowledge funding from the Advance Research Project of China Ordnance Equipment (Grant No. 51312050305), and Key Innovation Team for Magnetic Materials of Zhejiang Province and the China North Materials Science and Engineering Technology Group. 


\section{References}

[1] S. Yabukami, H. Mawatari, et al. J. Magn. Magn. Mater Vol. 290-291 (2005), p. 1318

[2] L.V. Panina, D.P. Makhnovskiya, K. Mahri, J. Magn. Magn. Mater Vol. 272-276 (2004), p. 1452

[3] E. Fernández, A.V. Svalov, et al. IEEE Trans. Magn. Vol. 18-21 (2013), p. 49

[4] H. Chiriac, N. Lupu, A.M. Fecioru, et al. J. Magn. Magn. Mater Vol. 290-291 (2005), p. 857

[5] Z. H. Zhang, B.Y. Li, W.L. Cui, J.X. Xie, J. Magn. Magn. Mater Vol. 323 (2011), p.1712

[6] S. Gudoshnikov, N. Usov, A. Nozdrin, et al. Phys. Status Solidi A Vol. 211 (2014), p. 980

[7] F.X. Qin, H.X. Peng, M.H. Phan, Mater. Sci. Engin. B Vol. 167 (2010),p.129

[8] H. Chiriaca, Maria Neagua, M. Vazquezb, et al. Magn. Magn. Mater. Vol. 251-253 (2002), p.242

[9] C.W. Liu, G.F. Sun, Z.F. Chen, et al. Mater. Devic Vol. 23-25 (2007) 4

[10]J.S. Liu, F.Y. Cao, D.W. Xing, et al. J. Alloys Compd Vol. 541 (2012), p. 215

[11]M. Coisson, P. Tiberto, F. Vinai, et al. J. Magn. Magn. Mater Vol. 510-514 (2008), p. 320

[12]H. Chiriac, M. Tibu, V. Dob, J. Magn. Magn. Mater Vol. 290-291 (2005), p.1142

[13]V. Zhukova, M. Ipatov, J. González, et al. IEEE Trans. Magn. Vol. 44 (2008) 3958-3961.

[14]V. Raposo, D. Garcia, M. Zazo, et al. J. Magn. Magn. Mater Vol. 272-276 (2004), p. 1463

[15]E. Costa Silva1, L.A.P. Gusmao, C.R. Hall Barbosa, et al. Sci. Technol 035204 (2011) 22

[16]M. ThanhTung, L. ThiThuHang, L. AnhTuan, et al. Physica B Vol. 16-20 (2014) 442

[17]X.Z. Zhou, G.H. Tu, H. Kunkel, G. Williams, Sens. Actuators A Vol. 387-392 (2006) 125.

[18]P. Tiberto, F. Vinai, O. Rampado, et al. J. Magn. Magn. Mater Vol. 196-197 (1999), p. 388

[19]A. Chizhik, M. Ipatov, A. Stupakiewicz, et al. Phys. Status Solidi C Vol. 11 (2014), p. 986

[20]M. Malátek, L. Kraus, Sens. Actuators A Vol. 16 (2010), p. 441

[21] V. Zhukova, P. Umnov, V. Molokanov, et al. IEEE Trans. Magn. Vol. 48 (2012), p.4034 